\title{
Evolution of Stability in a Cold-Active Enzyme Elicits Specificity Relaxation and Highlights Substrate-Related Effects on Temperature Adaptation
}

\author{
Pietro Gatti-Lafranconi ${ }^{1}$, Antonino Natalello ${ }^{1}$, Sascha Rehm ${ }^{2}$, \\ Silvia Maria Doglia ${ }^{1}$, Jürgen Pleiss ${ }^{2}$ and Marina Lotti ${ }^{1 *}$
}

${ }^{1}$ Department of Biotechnology and Biosciences, State

University of Milano-Bicocca,

Piazza della Scienza 2, I-20126

Milano, Italy

${ }^{2}$ Institute of Technical Biochemistry, University of Stuttgart, Allmandring 31, D-70569 Stuttgart, Germany

Received 25 August 2009; received in revised form 8 October 2009; accepted 14 October 2009

Available online

20 October 2009

\begin{abstract}
Molecular aspects of thermal adaptation of proteins were studied by following the co-evolution of temperature dependence, conformational stability, and substrate specificity in a cold-active lipase modified via directed evolution. We found that the evolution of kinetic stability was accompanied by a relaxation in substrate specificity. Moreover, temperature dependence and selectivity turned out to be mutually dependent. While the wild-type protein was strictly specific for short-chain triglycerides (C4) in the temperature range $10-50^{\circ} \mathrm{C}$ and displayed highest activity in the cold, its stabilized variant was able to accept C8 and C12 molecules and its selectivity was temperature dependent. We could not detect any improvement in the overall structural robustness of the mutant when the structure was challenged by temperature or chemical denaturants. There is, however, strong evidence for local stabilization effects in the active-site region provided by two independent approaches. Differential scanning fluorimetry revealed that the exposure of hydrophobic patches (as the active site is) precedes denaturation, and molecular dynamics simulations confirmed that stability was obtained by restriction of the mobility of the lid, a flexible structure that regulates the access to the enzyme active site and influences its stability. This reduction of lid movements is suggested to be accompanied by a concomitant increase in the mobility of other protein regions, thus accounting for the observed broadening of substrate specificity.
\end{abstract}

(C) 2009 Elsevier Ltd. All rights reserved.

Keywords: temperature adaptation; directed evolution; molecular dynamics; differential scanning fluorimetry; circular dichroism

\section{Introduction}

The application of biological concepts such as evolution, fitness, and adaptation to the study of specific molecular properties is strongly influencing

${ }^{*}$ Corresponding author. E-mail address: marina.lotti@unimib.it.

Present address: P. Gatti-Lafranconi, Department of Biochemistry, University of Cambridge, 80 Tennis Court Road, Cambridge CB2 1GA, UK.

Abbreviations used: DSF, differential scanning fluorimetry; $\mathrm{GuCl}$, guanidinium chloride; $\mathrm{MD}$, molecular dynamics; PFL, Pseudomonas fragi lipase; $T_{\mathrm{m}}$, melting temperature; RMSF, root-mean-square fluctuation; DMSO, dimethyl sulfoxide. the current approach of protein science. A number of novel issues build up the present frame of knowledge for investigating function and dynamics of proteins: flexibility as a key to enzyme activity, promiscuity as a source of selective advantages, ${ }^{1,2}$ the role of evolution and degeneration, ${ }^{3}$ and the definition of the active role of structural disorder, ${ }^{4}$ to mention a few of them.

This body of information prompts pathways of protein evolution to be investigated not only as for the emergence of specific features (i.e., stability, specificity, among others) but also, more interestingly, as concerns the shape of the pathway itself, that is, how (apparently) unrelated properties coexist and coevolve. ${ }^{1,5}$ As this kind of studies requires information about a large number of variants, the generation of diversity in the test tube by directed evolution is extremely powerful, since it 
also allows applying selective pressures that were never experienced in nature. Another genetic variability is possible, that is, isoenzymes and homologues of the same protein that can be isolated from nonconventional biological sources such as extremophilic or non-culturable organisms. ${ }^{6-8}$

In this work, we tackle some aspects of the evolution of thermal adaptation in enzymes by analyzing the interconnections between temperature dependence, conformational stability, and specificity in evolved enzyme variants. Fitness towards unusual and even extreme temperatures is a landmark of proteins from extremophiles, in which the ability to cope with challenging hot or cold environments is critical as temperature directly affects reaction kinetics, protein mobility, and structural flexibility. ${ }^{9}$ Here, subtle adjustments and buffering compensations in the protein structure have evolved to guarantee the correct balance between activity and stability. ${ }^{10}$ Cold-active enzymes have to face the challenge to stay active at temperatures at which catalysis is disfavored because of both thermodynamic and dynamic factors, related to reduced protein motions. ${ }^{11} \mathrm{~A}$ fine-tuning of the inherent flexibility of the protein polymer has been identified as the key to adaptation. $^{12}$ This view was substantiated by experimental and computational evidence and further corroborated by the observation that coldactive proteins share some similarities with intrinsically disordered proteins, ${ }^{4}$ which are, by definition, the most flexible polypeptide chains. As a consequence, structural disorder has been proposed as a possible driving force of adaptation to the cold. ${ }^{13}$ It was also argued that, rather than increasing flexibility, enzymes might reduce the reaction activation barrier by developing active sites that require less energy to reorganize the polar and ionized groups that stabilize the transition state. ${ }^{14}$ This viewpoint is intriguing and it suggests that changes in the interaction network of the active site might have been crucial in the evolution of fitness in the cold (and temperature adaptation in general), and this strategy is likely to happen through mutation of distal, not essential, and poorly conserved amino acids.

Enhanced structural flexibility easily produces heat instability, and this is the case of most coldactive enzymes. However, in several cases, increased conformational flexibility is restricted to the polypeptide regions around the active site and does not extend to the whole protein structure. Thus, in principle, there is no need for a cold-active enzyme to be thermolabile. ${ }^{15-18}$ Nevertheless, this is a common problem and a number of directed evolution studies were devoted to increasing the optimal temperature and the heat stability of cold-active enzymes or to lowering that of (hyper)thermophiles both for knowledge and for application purposes. ${ }^{19-23} \mathrm{~A}$ remarkable outcome of these studies was that temperature adaptation and heat stability are partially independent and can be evolved separately, with the most recurrent combination (increased cold activity/loss in thermal stability) being only the easiest way to fulfill selective pressure requirements.

Generally speaking, the development by directed evolution of new properties, be they specificity, specific activity, or a new catalytic activity, is assumed to impair the protein thermodynamic stability, mainly because most mutations are destabilizing, in particular if located in or near the active site. This observation prompted the concept that enzymes have to overcome a stability constrain at least at some points of the pathway to acquire new properties (i.e., to adapt). ${ }^{24,25}$ Though this trade-off between stability and function can be hardly generalized, at least with our present knowledge, it points once more to a complex role of temperature in shaping the evolution of enzymes and proteins and suggests that the study of the effects of random evolution on thermo-adapted enzymes is a good tool for defining the limits of these concepts.

We take here the particular case of a cold-active lipase to analyze the relationships between thermal adaptation and other enzyme properties. Lipases catalyze the hydrolysis of triglycerides and other substrates containing ester bonds and are broadly diffused in nature. ${ }^{26,27}$ Lipases are of interest in this context also because their activity is regulated by mobile loop structures that build a kind of lid onto the active site and are inherently flexible. The same structure couples activity with specificity in that it not only regulates access to the active site but also is part of it and interacts with substrate molecules. In previous papers, we observed that the evolution of thermal stability in the Pseudomonas fragi lipase (PFL) resulted in variants in which temperature optimum $\left(T_{\text {opt }}\right)$ and structure stability were uncoupled, in the sense that the increase in $T_{\text {opt }}$ and kinetic stability appeared to be not paralleled by any increase in conformational robustness. ${ }^{28}$ In this work, we compare the wild-type protein and the "better adapted" mutant by biochemical, biophysical, and computational methods and show that subtle movements of localized and scarcely structured protein regions do account for some of the evolved properties. Moreover, we found that, in evolved lipases, temperature adaptation is strongly substrate dependent. The different approaches we are here reporting scratch a complex picture where specificity, stability, and thermal adaptation combine to generate the most efficient catalyst in the specific condition set by the selection pressure applied.

\section{Results}

\section{The temperature dependence of lipase activity varies with the substrate}

Proteins used in this study are derived from a cold-active, temperature-sensitive lipase cloned from the psychrotrophic bacterium P. fragi (PFL). The PFL structure is predicted to conform to the $\alpha$ / $\beta$-hydrolase fold common to all lipases and to bear 
a mobile "lid" structure onto the active-site crevice. $^{29}$ In previous work, this sequence was evolved in vitro to increase its temperature stability without compromising cold activity. The bestperforming variant obtained (DE-2-4) was 5-fold more stable than the wild type at $42{ }^{\circ} \mathrm{C}$ and displayed improved specific activity in the temperature range $29-42{ }^{\circ} \mathrm{C}$ with a shift in $T_{\text {opt }}$ from 29 to $42{ }^{\circ} \mathrm{C} .{ }^{28}$ This protein carries four amino acid substitutions (D2Y, H30N, R127G, and R152G) that map at the very $\mathrm{N}$-terminus (position 2), in the central $\beta$-sheet (position 30), and in the lid region (positions 127 and 152) (Fig. 1). While the stabilizing effect of the substitutions in the lid is not surprising, particularly because they remove exposed arginine residues, and position 2 is located in the N-terminal flexible tail close to the histidine tag and does not interact with the core protein structure, the contribution of the amino acid at position 30 can hardly be predicted on rational bases. The role of this residue in fact is not obvious, as it is located in a cluster of hydrophobic and aromatic residues that has no correspondence in any other known lipase. Therefore, we produced a PFL with the lone H30N replacement. The biochemical and conformational properties of the wildtype PFL, of the mutant DE-2-4, and, wherever appropriate, of this intermediate variant protein are analyzed in-depth and compared with the aim of understanding their interconnections (if any exist).
As already mentioned, cold activity has been related to the flexibility of the enzyme's active site and its surrounding. ${ }^{18}$ Therefore, we speculated that changes in temperature dependence and kinetic stability observed in the evolved lipases-although obtained through the substitution of amino acids that map far away from the active site-might cause subtle changes in this region, and thus, we tested the hydrolysis of different substrates. In Fig. 2a, we report the activity profile of wild-type, H30N, and DE-2-4 lipases measured in the temperature range 10-50 ${ }^{\circ} \mathrm{C}$ on triglycerides with different chain lengths, which are tributyrrin (C4), tricaprylin (C8), and trilaurin (C12). Wild-type PFL showed a preference for the shortest chained substrate at any tested temperature, whereas the $\mathrm{H} 30 \mathrm{~N}$ protein was rather unspecific in the cold and shifted towards the C4 substrate only above $29{ }^{\circ} \mathrm{C}$. The DE-2-4 lipase was always more active on the $\mathrm{C} 8$ triglyceride but its selectivity decreased with temperature with a rise in activity towards both $\mathrm{C} 4$ and $\mathrm{C} 12$. This progressive relaxation of specificity suggests the occurrence of rearrangements in the active-site region. Accordingly, the activity of the lipase DE-2-4 but not of the wild-type enzyme was affected by the long-chain inhibitor Orlistat (Table 1). The optimal temperature of the wild type was $29^{\circ} \mathrm{C}$ on both substrates, while the optimum for DE-2-4 was $42{ }^{\circ} \mathrm{C}$ on $\mathrm{C} 4$ and $\mathrm{C} 8$ substrates and $50{ }^{\circ} \mathrm{C}$ on trilaurin (C12). Interestingly, H30N displayed highest activity on C8 and C4 at 29

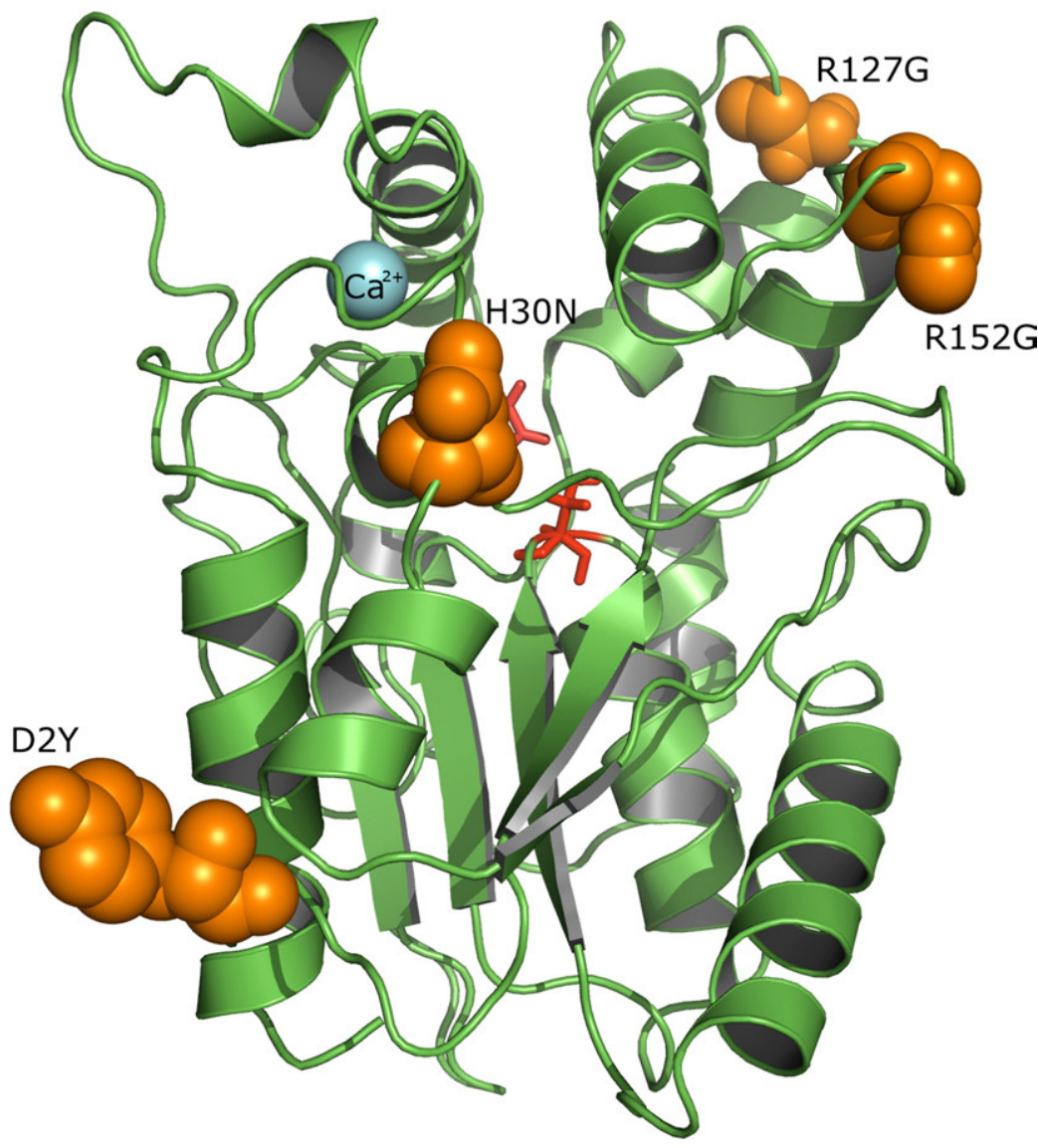

Fig. 1. PFL homology model. Residues mutated in DE-2-4 are colored orange and displayed as space fills, the catalytic triad is shown in red sticks, and the calcium ion is displayed as a light blue sphere. Residues are numbered according to the text. 
(a)

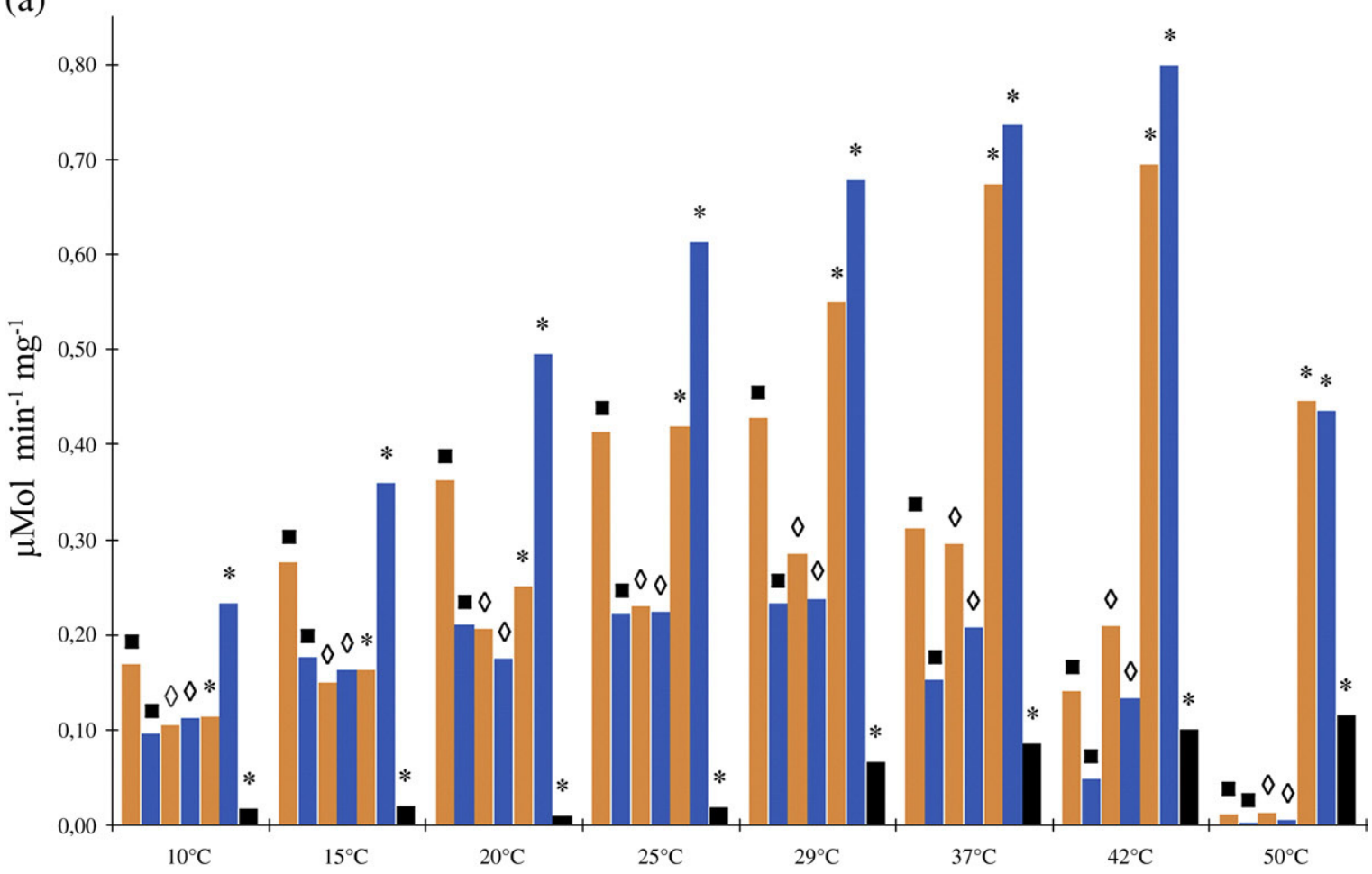

(b)

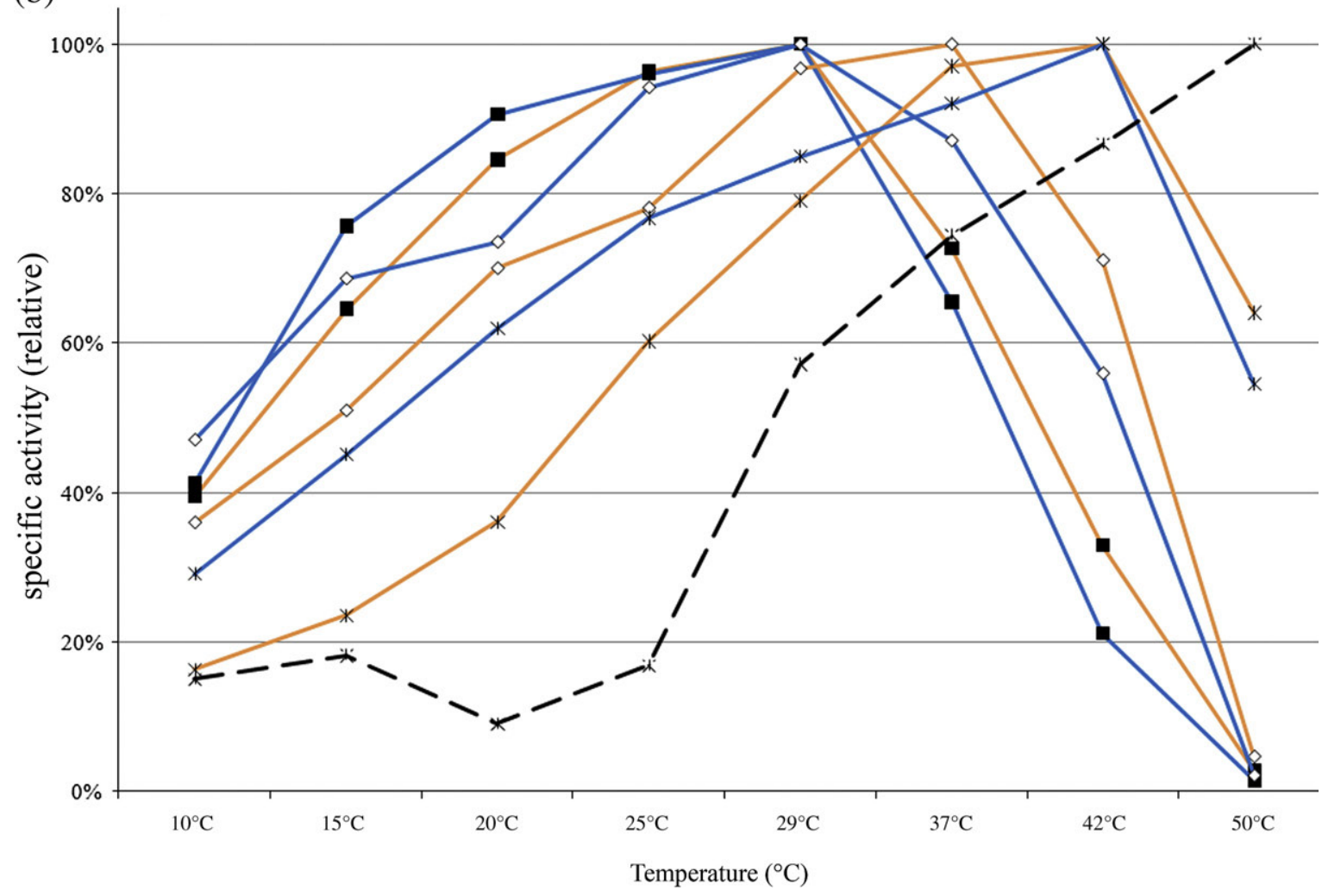

Fig. 2. (a) Specific activity of tested enzymes at the indicated temperatures. Symbols: $\mathbf{\square}$, wild type; $\diamond, H 30 \mathrm{~N}$; *, DE-2-4. Orange bars indicate tributyrin (C4), blue bars denote tricaprylin (C8), and black bars indicate trilaurin (C12). Standard deviation was below $10 \%$. Activity on $\mathrm{C} 12$ is only significant above $25^{\circ} \mathrm{C}$ due to poor solubility of the substrate at lower temperatures. (b) Relative activity of data reported in (a). Symbols and colors are as in (a) (C12 is indicated by a broken line). 
Table 1. Inhibition assay with Orlistat

\begin{tabular}{lcc}
\hline Enzyme & Molar excess, 226 (\%) & Molar excess, $566(\%)$ \\
\hline Wild type & 70 & 55 \\
DE-2-4 & 20 & 3 \\
\hline
\end{tabular}

Relative activity after $15 \mathrm{~min}$ of incubation with the inhibitor (referred to an untreated sample) in the presence of different molar excess of Orlistat. Experiments were repeated in duplicate. Enzymes incubated in pure buffer showed no activity loss while $5 \%$ DMSO (corresponding to the volume of Orlistat added for a molar excess of 566) caused $20-30 \%$ activity reduction.

and at $37{ }^{\circ} \mathrm{C}$, respectively (Fig. 2b). If, on the medium-length substrate tricaprylin (C8), DE-2-4 was the best catalyst at any tested temperature with a 3-fold increase in specific activity in the whole considered range, this was not the case on the shorter substrate. Indeed, the wild-type lipase turned out to be the best-performing cold-adapted tributyrin hydrolase, since it displayed the highest activity on $\mathrm{C} 4$ between 10 and $25^{\circ} \mathrm{C}$. This first set of data drew our attention on a further parameter to be considered: substrate as a key player in the temperature dependence of our enzymes.

In an attempt to answer the question of whether and how specificity and temperature dependence coevolved, we calculated from the data expressed in Fig. 2 the C8/C4 activity ratio at all temperatures considered (not shown). Such a ratio is constant for the wild type, whereas mutants perform better on tricaprylin (C8) at low temperature only. In other words, changes in selectivity in the evolved proteins become apparent only in the cold. For the sake of clarity, we should recall that parameters applied for mutant screening were improved heat stability and retained cold activity. For each substrate/enzyme pair, we defined a cold-adaptation index $\left(\mathrm{CA}_{\mathrm{i}}\right)$ calculated from the ratio between activity at $10{ }^{\circ} \mathrm{C}$ and activity at the optimal temperature (Table 2). $\mathrm{H} 30 \mathrm{~N}$ was found to perform better in the cold on C8 and to retain a similar ratio on $C 4$ despite the increase in $T_{\text {opt }}$. On the other hand, DE-2-4 maintained a $\mathrm{CA}_{\mathrm{i}}$ consistent with cold adaptation only on its preferred substrate (C8). The fact that DE-2-4 lost cold activity on $\mathrm{C} 4$ but evolved it on $\mathrm{C} 8$ supports the concept of specialization as a key to evolution: the most critical property - in this case, cold activitywas retained, but on a different substrate. Calculated activation energies (Table 2) indicate that both mutants possess higher $E_{\mathrm{a}}$ values in the range 15$29{ }^{\circ} \mathrm{C}$, but when the same analysis is extended to their temperature optimum, activation energies on the preferred substrate decrease significantly, approaching values measured for the wild type.

\section{Local stabilization of the protein structure accounts for changes in temperature dependence}

Preliminary measurements showed that differences in kinetic stability and $T_{\text {opt }}$ of lipase DE-2-4 with respect to the wild type do not depend on major structural rearrangements that would result in changes in conformational stability. ${ }^{28}$ To gain a deeper insight into the relationships between structural robustness and temperature dependence, we probed the wild type and the mutant protein with temperature, chemical denaturants, and specific dyes and tracked conformational changes by spectroscopic and fluorimetric methods. This analysis was particularly tricky because of the intrinsic lability of the wild-type PFL to standard handling, concentration, and storage procedures, which also accounts for the low quality of its spectral response. Comparison of circular dichroism (CD) spectra of freshly purified proteins at $20^{\circ} \mathrm{C}$ did not reveal any major difference in the secondary-structure content of the two enzymes (Fig. 3a). Heating the protein solutions from 20 to $100{ }^{\circ} \mathrm{C}$ produced a similar loss in ellipticity in both samples, and all CD spectra were almost identical. This effect was irreversible as cooling back to $20^{\circ} \mathrm{C}$ did not result in the formation of secondary structure. The reduction of the signal recorded in particular above $42{ }^{\circ} \mathrm{C}$ was homogenous at all wavelengths, and this observation was suggestive of the occurrence of protein aggregation due to the initial protein unfolding (Fig. $3 b$ and $c$ ). To limit the extent of aggregation during the acquisition time, we performed a rapid scan at $222 \mathrm{~nm}$ in the temperature range $20-100{ }^{\circ} \mathrm{C}$. Quite surprisingly, the wild-type protein displayed a $T_{\mathrm{m}}$ slightly higher than that of the mutant (Fig. 4 and Table 3). This result rules out the hypothesis that the higher temperature optimum of the evolved mutant may be a consequence of an increased overall thermal stability of its structure (Fig. 4). Further insight was provided by the evaluation of the HT or dynode values $^{30}$ that relate to changes in the turbidity of the solution, that is, to protein aggregation and precipitation (Fig. 4, inset). The increase in this value observed above $40{ }^{\circ} \mathrm{C}$ indicates an ongoing aggregation process, while its reduction

Table 2. Activation energy and cold-adaptation index

\begin{tabular}{|c|c|c|c|c|c|c|}
\hline \multirow{2}{*}{\multicolumn{2}{|c|}{$\begin{array}{c}\text { Optimal } \\
\text { temperature } \\
\left({ }^{\circ} \mathrm{C}\right)\end{array}$}} & \multirow[b]{2}{*}{$\mathrm{CA}_{\mathrm{i}}$} & \multicolumn{2}{|c|}{$15-29$} & \multicolumn{2}{|c|}{ 15-optimum } \\
\hline & & & $\begin{array}{c}E_{\mathrm{a}} \\
\left(\mathrm{kJ} \mathrm{mol}^{-1}\right)\end{array}$ & $R^{2}$ & $\begin{array}{c}E_{\mathrm{a}} \\
\left(\mathrm{kJ} \mathrm{mol}^{-1}\right)\end{array}$ & $R^{2}$ \\
\hline \multicolumn{7}{|c|}{ Wild type } \\
\hline C4 & 29 & 0.39 & 22.59 & 0.9189 & & \\
\hline \multicolumn{7}{|l|}{ H3ON } \\
\hline C4 & 37 & 0.36 & 31.25 & 0.9681 & 22.72 & 0.8899 \\
\hline $\mathrm{C} 8$ & 29 & 0.47 & 21.21 & 0.9412 & 21.21 & 0.9412 \\
\hline \multicolumn{7}{|c|}{$D E-2-4$} \\
\hline C4 & 42 & 0.16 & 63.87 & 0.9961 & 40.91 & 0.9022 \\
\hline C8 & 42 & 0.29 & 32.88 & 0.9733 & 20.64 & 0.8902 \\
\hline C12 & 50 & 0.15 & - & - & 52.26 & 0.7811 \\
\hline
\end{tabular}

Activation energy parameters for the three enzymes in different temperature ranges are shown (correlation coefficient at any interval is also reported). The $15-29^{\circ} \mathrm{C}$ range is used as reference (it being the range of stability for the wild-type enzyme). Optimal temperature of each enzyme/substrate pair is indicated. $E_{\mathrm{a}}$, activation energy; $R^{2}$, correlation coefficient; $C \mathrm{~A}_{\mathrm{i}}$, cold-adaptation index (calculated as the activity at $10{ }^{\circ} \mathrm{C} /$ activity at $T_{\text {opt }}$ ratio). 

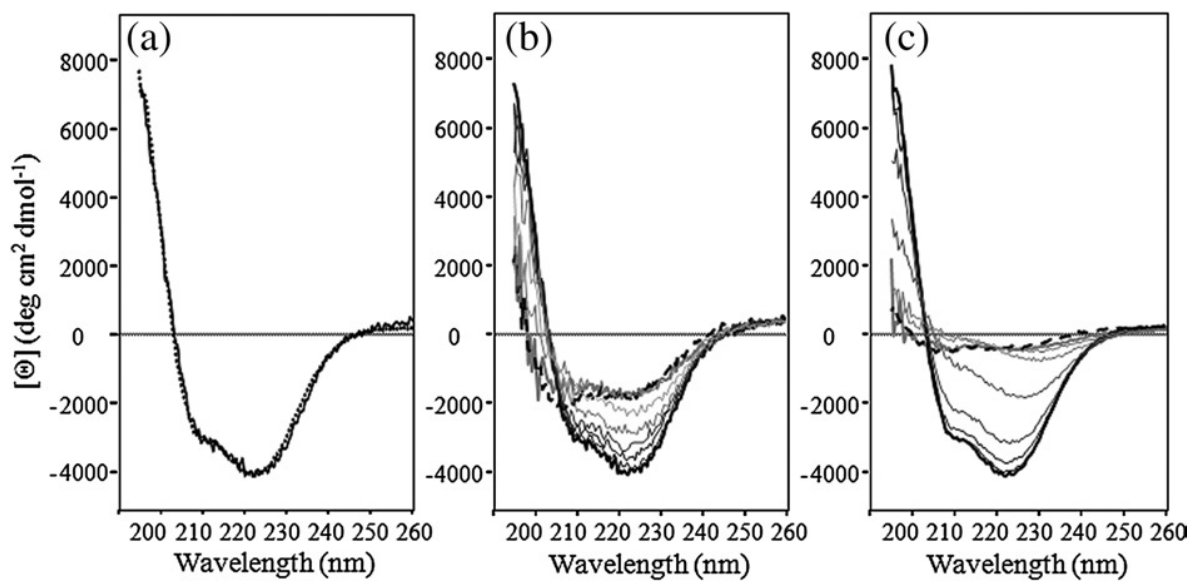

Fig. 3. CD spectra of wild type and DE-2-4 in the 195- to 250-nm range. (a) Normalized CD spectra of wild type (continuous line) and DE-2-4 (dotted line) recorded at $20^{\circ} \mathrm{C}$. (b) $\mathrm{CD}$ spectra of wild type from 20 to $100{ }^{\circ} \mathrm{C}$. (c) $\mathrm{CD}$ spectra of DE-2-4 from 20 to $100^{\circ} \mathrm{C}$. In both (b) and (c), line code goes from black $\left(20^{\circ} \mathrm{C}\right)$ to gray $\left(100^{\circ} \mathrm{C}\right)$ as temperature increases, while black broken lines indicate spectra recorded after cooling back the solution at $20^{\circ} \mathrm{C}$.

above $60{ }^{\circ} \mathrm{C}$ reports on precipitation. This could be explained in terms of small rearrangements triggered by relatively low temperatures (around $40^{\circ} \mathrm{C}$ ) that would induce proteins to aggregate in soluble assemblies that subsequently precipitate at higher temperatures.

Further experiments were performed in order to monitor the early unfolding steps in the lipase DE-24 by measuring changes in the $222-\mathrm{nm}$ band of the protein solution upon rapid heating from 20 up to 42 or $50{ }^{\circ} \mathrm{C}$ (Fig. 5). While the structure was relatively stable for the first $10 \mathrm{~min}$ at $42{ }^{\circ} \mathrm{C}$, it underwent a very rapid unfolding process a few seconds after reaching $50{ }^{\circ} \mathrm{C}$. HT values indicated that unfolding is not followed by precipitation. This observation is consistent with the occurrence of minor rearrangements in the critical temperature values 42 and $50{ }^{\circ} \mathrm{C}$, leading to aggregation and possibly to an impairment in activity even though complete unfolding and precipitation only occur at higher temperatures.

Thermal adaptation is considered a consequence of selective pressure (or of its absence), but is stability against other denaturants affected? We explored this issue by testing our enzymes for robustness towards chemical denaturation by exposing them for $10 \mathrm{~min}$ to growing concentrations of guanidinium chloride $(\mathrm{GuCl})$. Fluorescence spectra (Fig. 6) did not reveal any appreciable change in the intensity or peak position up to $3 \mathrm{M} \mathrm{GuCl}$, whereas a shift in the peak position was evident at $6 \mathrm{M} \mathrm{GuCl}$, when the protein is unfolded. ${ }^{31}$ Despite its low thermal stability, the lipase structure appears to be quite robust towards chemical denaturation. Interestingly, $1 \mathrm{~h}$ incubation with low concentration of denaturant $(0.1 \mathrm{M} \mathrm{GuCl})$, while not affecting peak position, induced a reduction of about $25 \%$ in the signal intensity (Fig. 6, inset). This can be accounted for by events of precipitation rather than by changes in the exposure of tryptophan and reinforce the hypothesis that local, small structural rearrangements are the driving force for PFL unfolding.

It was therefore interesting to monitor possible structural changes localized at the active site. To this end, we performed differential scanning fluorimetry $(\mathrm{DSF})^{32,33}$ measurements in the presence of SYPRO

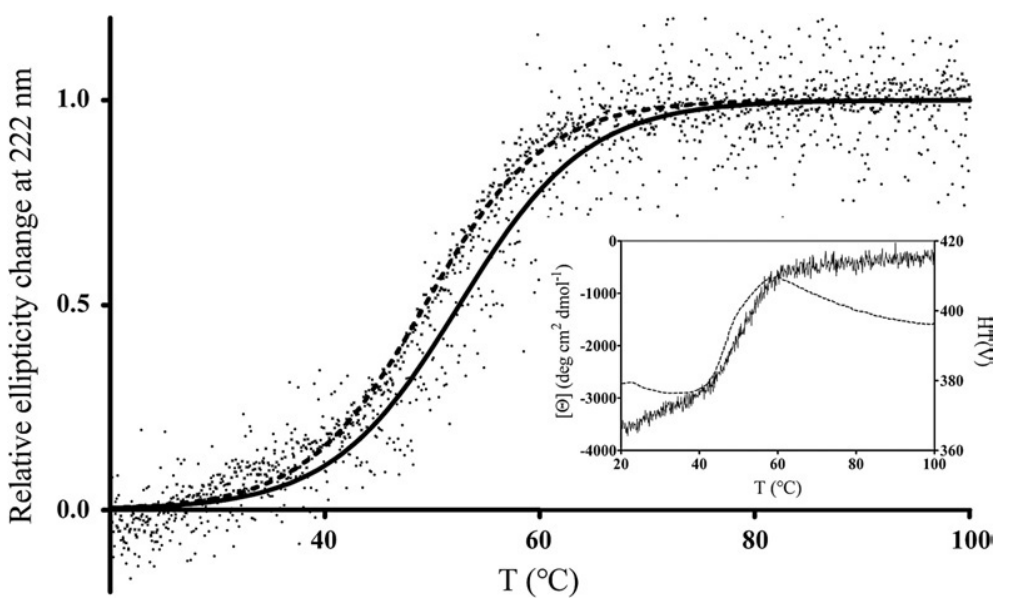

Fig. 4. Relative ellipticity change for wild type (black line) and DE2-4 (broken line) registered at $222 \mathrm{~nm}$ upon heating. Inset: ellipticity and $\mathrm{HT}(V)$ plots for DE-2-4. $R^{2}$ values are 0.93 and 0.99 for wild type and mutant, respectively. 
Table 3. Melting temperatures

\begin{tabular}{lccc}
\hline & $\mathrm{CD}\left({ }^{\circ} \mathrm{C}\right)$ & $\mathrm{DSF}\left({ }^{\circ} \mathrm{C}\right)$ & Difference $\left({ }^{\circ} \mathrm{C}\right)$ \\
\hline Wild type & $52.5 \pm 0.6$ & $42.6 \pm 1.2$ & $9.9 \pm 1.3$ \\
DE-2-4 & $49.0 \pm 0.2$ & $46.2 \pm 0.2$ & $2.8 \pm 0.3$ \\
\hline
\end{tabular}

Melting temperatures measured for wild type and DE-2-4 enzymes from CD or DSF. Difference between the two values is also reported for both enzymes.

Orange, a fluorescent dye that specifically binds to hydrophobic patches. In the wild-type lipase, $T_{\mathrm{m}}$ values determined by DSF are lower than those determined by $C D$, indicating that exposure of hydrophobic patches anticipates secondary-structure unfolding (Table 3). Due to its high hydrophobicity and lack of secondary structure, the active site is likely to be responsible for this discrepancy. In this light, the higher $T_{\mathrm{m}}$ value obtained for mutant DE-24 and the reduction in the difference between the two $T_{\mathrm{m}}$ values (measured with DSF or CD, Table 3 ) suggest that this variant is locally stabilized at the active site or in its proximity.

The interpretation of DSF data is supported by molecular dynamics (MD) simulations (see next paragraph) and by the observation that $T_{\text {opt }}$, and not secondary-structure stability $\left(T_{\mathrm{m}}\right.$ from $\left.C D\right)$, is increased in the mutant. The stabilization effect accounts for its higher kinetic stability but is not sufficient to significantly have an impact on the robustness of the overall structure.

\section{MD simulations}

The models needed for the MD simulations were built based on a sequence alignment with the homologous Burkholderia glumae lipase (see Materials and Methods for details) and allowed simulations to be carried out on the wild type and DE-2-4 mutant of PFL. Plotting root-mean-square fluctuation (RMSF) values against sequence position provides indications on the extent and localization of structure movements. Although absolute temperature values in MD simulations do not precisely reflect experimental temperatures, relative differences are consistent and allow for a comparison of the behavior of the two proteins from low $\left(15^{\circ} \mathrm{C}\right)$ to high $\left(50{ }^{\circ} \mathrm{C}\right)$ temperature. Data reported in Fig. 7 highlighted some significant properties of the system: (1) compared to the wild type, the mutant shows a similar overall profile, with changes in RMSF being localized around specific regions rather than dispersed across the whole sequence space; (2) even though mutations $\mathrm{R} 127 \mathrm{G}$ and $\mathrm{R} 152 \mathrm{G}$ reduce the mobility of the lid, several other regions are more flexible in the mutant; (3) in the two enzymes, different regions are endowed with different degrees of mobility, probably as a result of longrange interactions (regions 20-30 and 230-240 for the mutant and regions 65-75, 125-130, and 205-210 in the wild type). With all due caution, MD results support experimental data in providing proof that induced structural changes are localized and of moderate intensity. Moreover, they suggest that regions other than the active site are involved in changes in the temperature-dependent catalytic behavior, such as the 20-30 loop at one end of the active-site cleft (which possibly anchors part of the lid to the structure beneath) or the calciumcoordinating region (residues 200-210). The most significant changes in flexibility were identified at position 129. This residue is part of one of the flexible hinges connecting the lid helix and the rest of the protein. While the DE-2-4 mutant shows constant flexibility from 15 to $45^{\circ} \mathrm{C}$, the wild type increases flexibility above $30^{\circ} \mathrm{C}$ (Fig. 8). Plotting of RMSF changes at position 129 over temperatures indicates that higher mobility correlates well with the loss of activity of the wild type, since DE-2-4 shows smaller changes in both parameters and lower correlation (Fig. 9). Plotting of RMSF values from other protein regions (position 22, 70, 207, or 237) gave no correlation (not shown). This indicates that although the main cause for activity impairment in the wild type is lid mobility, reduction of its movements is achieved by increasing mobility of other regions, that is, by the effect of distal mutations.

\section{Discussion}

Comparison of the wild-type lipase to the evolved variant DE-2-4 provides evidence that the increase in kinetic stability results from complex adaptation events rather than from a generalized stabilization of the overall structure. Both samples in fact underwent similar processes of unfolding upon heat or chemical treatment. Only a detailed analysis of kinetics parameters, the use of DSF, and MD simulations disclosed subtle local differences. MD simulations, besides supporting the contribution to stability of constraints in the lid movements, provided novel insight into the unexpected broadening of the range of substrates accepted by the mutant that we hypothesize to be related to the increase in mobility of distal protein regions.

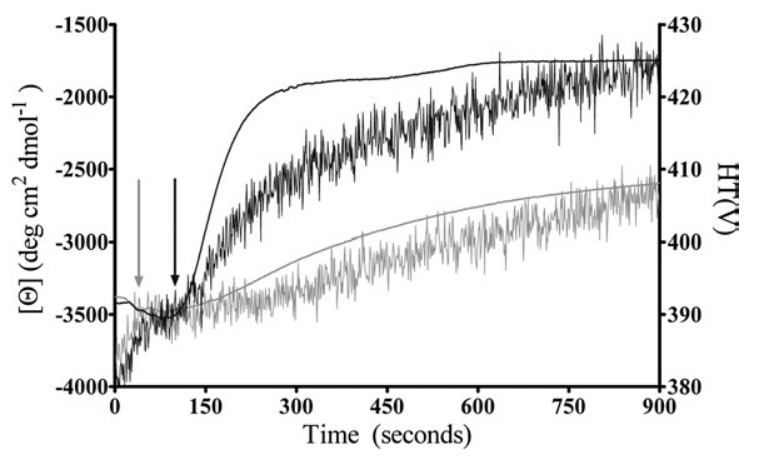

Fig. 5. Rapid kinetics of thermal unfolding for DE-2-4. Ellipticity at $222 \mathrm{~nm}$ and $\mathrm{HT}(V)$ values were recorded while rapidly heating samples from 20 up to $42{ }^{\circ} \mathrm{C}$ (gray) or $50{ }^{\circ} \mathrm{C}$ (black). Arrows indicate when the target temperature was reached (the same color code is used). 


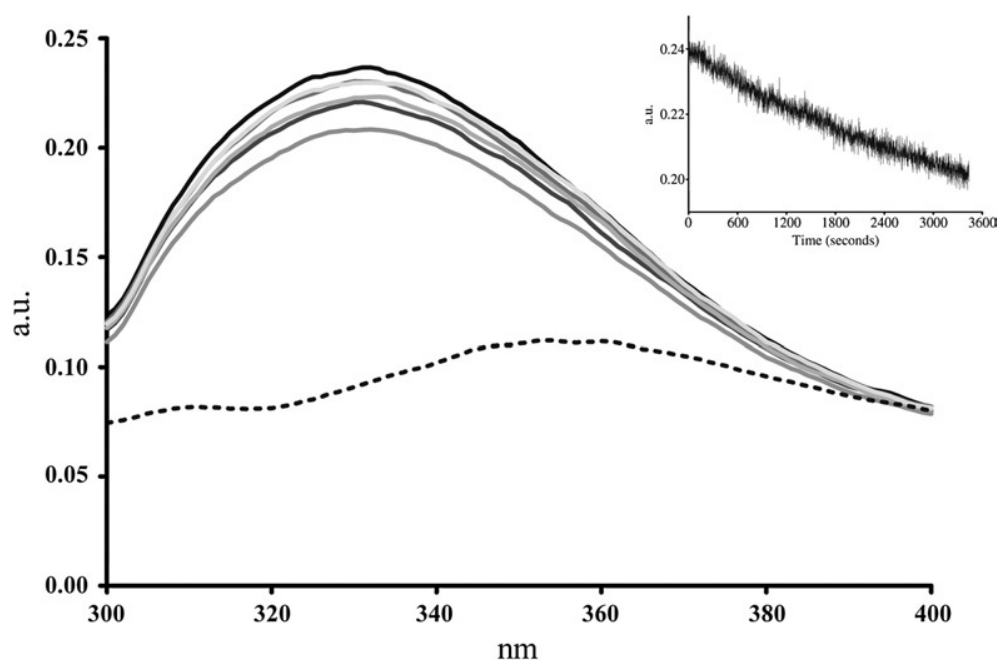

Fig. 6. Fluorescence measurements of wild-type PFL treated with different $\mathrm{GuCl}$ concentrations $(0,0.1,0.25,0.5,1.5$, and $3 \mathrm{M}$, from black to light gray lines, and $6 \mathrm{M}$, black broken line). Samples were excited at $280 \mathrm{~nm}$, and emission spectra were recorded in the 300- to 400-nm range. Measurements were performed right after mixing, allowing the solution to reach homogeneity but preventing effects due to longer incubations. Inset: changes in the 328-nm fluorescence band when the enzyme is incubated with $0.1 \mathrm{M} \mathrm{GuCl}$ for up to $1 \mathrm{~h}$. Chemical denaturation of DE-2-4 gave similar profiles.

Although our study does not extend that far in the assay of substrates, it is tempting to describe this relaxation of selectivity as increased promiscuity. ${ }^{34}$ This would be consistent with observations by other laboratories that stability supports evolvability and also that nonspecialized proteins might be intermediates in the pathways of evolution., ${ }^{3,35}$ It might be argued that we focused on a protein where selectivity and stability are naturally coupled through the lid structure. The lid in fact may be a nucleation focus for unfolding due to its flexibility, and on the other hand, in the open enzyme conformation, it builds a wall of the substrate binding site and is therefore of importance for substrate selection. Taking this peculiarity into account, the question remains: did we really evolve

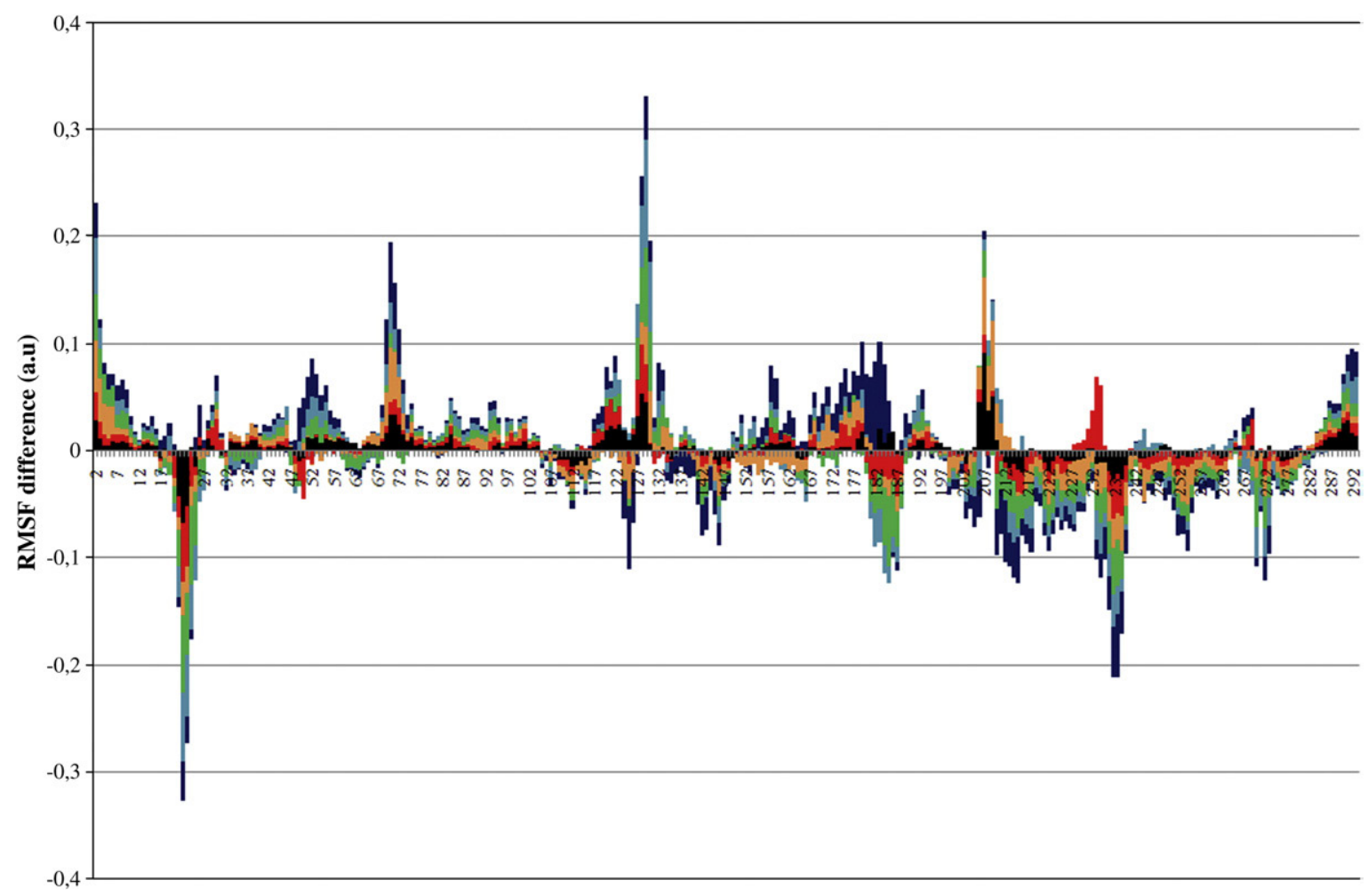

Fig. 7. RMSF difference (PFL minus DE-2-4) plotted against protein sequence. Changes are indicated with a different color for each tested temperature $\left(15^{\circ} \mathrm{C}\right.$, black; $25^{\circ} \mathrm{C}$, red; $35^{\circ} \mathrm{C}$, orange; $40^{\circ} \mathrm{C}$, green; $45^{\circ} \mathrm{C}$, light blue; $50{ }^{\circ} \mathrm{C}$, dark blue). 
$25^{\circ} \mathrm{C}$

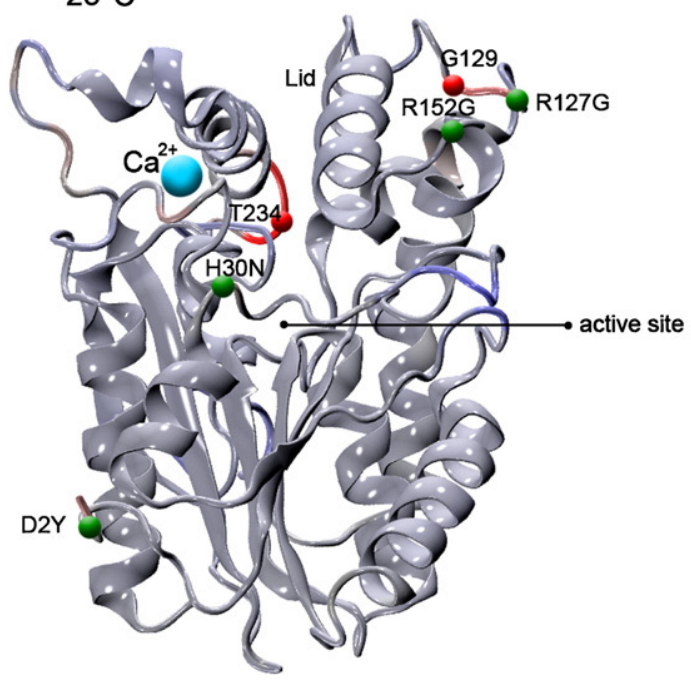

$45^{\circ} \mathrm{C}$

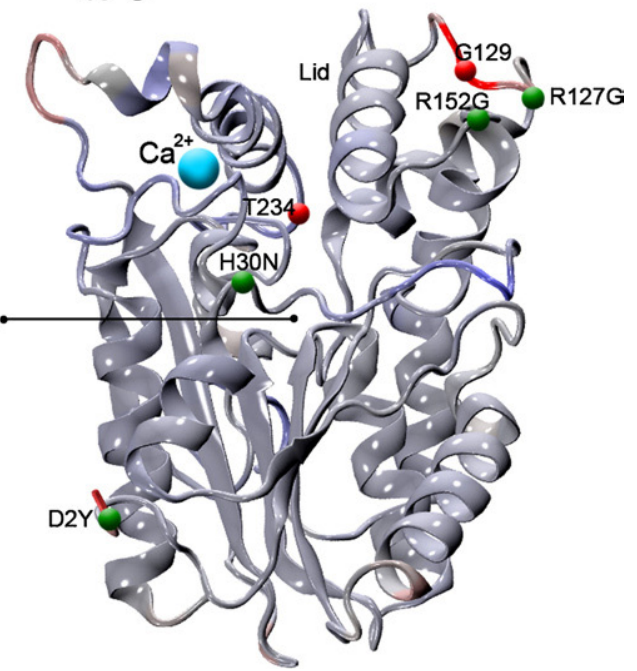

Fig. 8. Difference in flexibility between PFL wild type and mutant DE-2-4, averaged over two simulations and mapped on the homology model, simulated at $25^{\circ} \mathrm{C}$ (left) and $45^{\circ} \mathrm{C}$ (right). Color code: decrease and increase in the flexibility of DE-2-4 (as compared to that in the wild type) are denoted in dark red and dark blue, respectively; no change in flexibility is indicated in gray. At $45^{\circ} \mathrm{C}$, position 129 shows a significant decrease in flexibility in DE-2-4 (red). Residues mutated in DE-2-4 are colored green; the calcium ion is in light blue. Residues are numbered according to the text.

a more generalist protein (both for temperature dependence and for specificity)? Can this observation be embedded in the body of information about protein evolution? Apparently, this may be the case. The two proteins used in this study differ in a fundamental property. The wild-type PFL appears to fit a strict definition of cold-active tributyrin hydrolase. It is constrained both in the range of temperature where it is active and in the spectrum of substrates. On the contrary, selectivity depends on the temperature in the mutant (which also accepts larger substrates). This observation was, at first glance, very puzzling but found a possible explanation in the MD results suggesting a relaxation of a

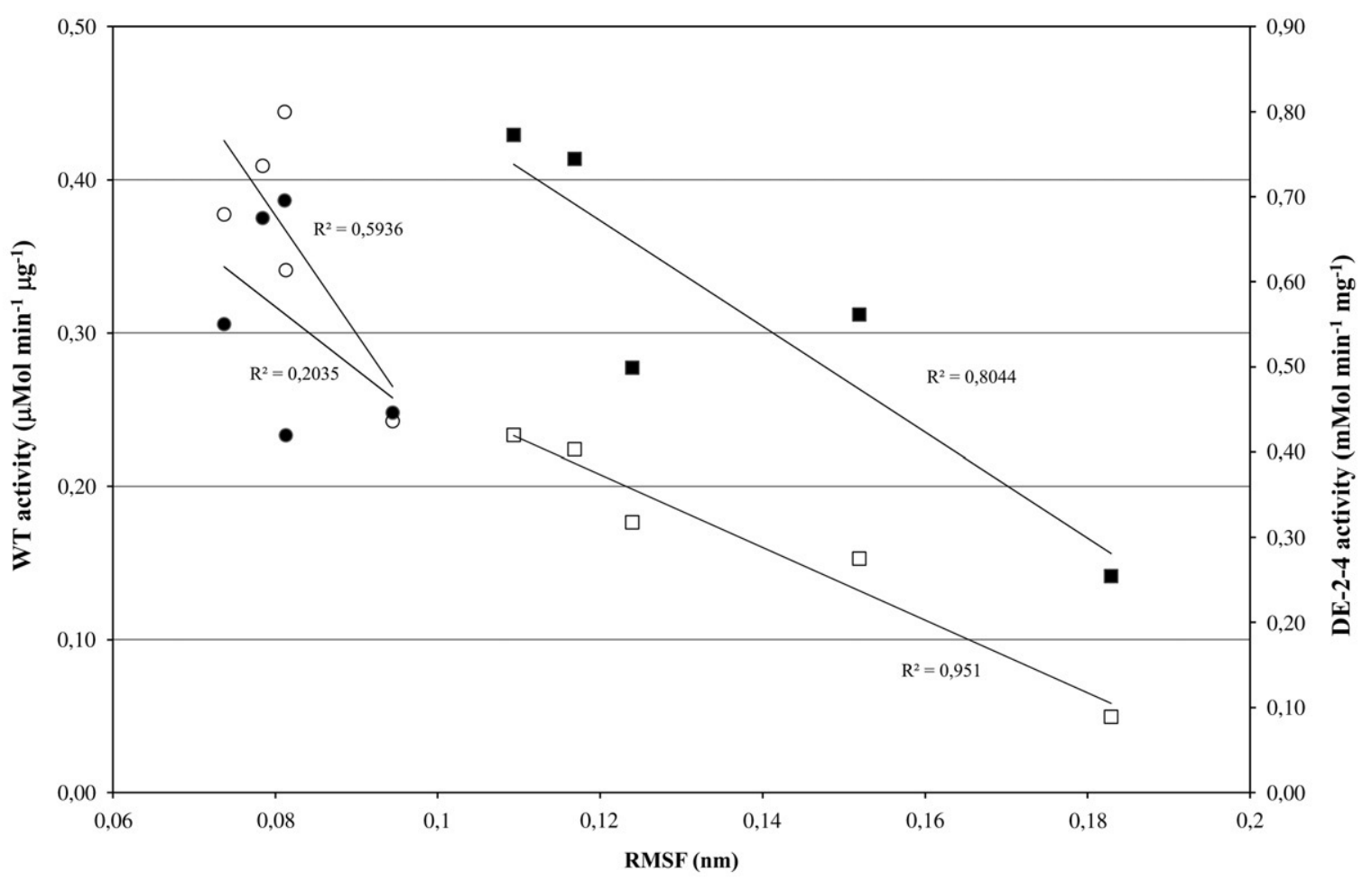

Fig. 9. Enzyme activity at different temperatures plotted against RMSF changes at position 129. Activities for wild type (squares) and DE-2-4 (circles) on C4 and C8 substrates (filled and open symbols, respectively) are reported. For clarity, correlation coefficients are indicated on the picture. 
few protein regions concomitant to the lid stabilization. Thus, coupling of stability and specificity can be rationalized by long-range effects acting differentially on different parts of the enzyme molecule. The interpretation of the temperature dependence of substrate specificity finds some support in a recently drawn theory ${ }^{5}$ proposing that proteins may fluctuate between different conformations, the relative frequencies of which would depend on their stability.

\section{Materials and Methods}

\section{Chemicals, strains, and fermentations}

If not otherwise stated, all chemicals and products were purchased from Sigma-Aldrich (USA) at analytical grade. Escherichia coli strain DH5a was used for standard cloning procedures while the BL21 (DE3) strain (Invitrogen, USA) was used for protein expression. All sequences were cloned in a pET19b plasmid (Novagen, USA). Standard growth medium was $0.5 \% \mathrm{NaCl}$ Luria broth (LB) supplemented with $100 \mu \mathrm{g} / \mathrm{ml}$ ampicillin (LB-amp) and agar $(18 \mathrm{~g} / \mathrm{L})$, when required. Protein production was carried out as follows: overnight cultures derived from single colonies were used to inoculate $300 \mathrm{~mL}$ of LB-amp; cultures were grown at $37^{\circ} \mathrm{C}$ until $\mathrm{OD}_{600}$ (optical density at $600 \mathrm{~nm}$ ) reached 0.4 and then transferred to $17^{\circ} \mathrm{C}$ for $45 \mathrm{~min}$; finally, they were induced with $0.1 \mathrm{mM}$ IPTG. Cells were harvested after $18 \mathrm{~h}$, and proteins were extracted and purified by IMAC as reported previously. ${ }^{28}$

\section{DNA manipulations}

Mutagenesis was performed on a pET19b plasmid (Novagen) bearing the wild-type PFL or mutant sequences fused at the N-terminus with a $\mathrm{His}_{6}$ tag. The $\mathrm{H} 30 \mathrm{~N}$ replacement was introduced by PCR using the following primers: H30N_for, 5'-CGCATCACTACTTTAATGGTATCAAGCAAGC-3'; H30N_rev, 5'-GCTTGCTTGATACCATTAAAGTAGTGATGCG-3'. PCR was performed in a final volume of $20 \mu \mathrm{l}$ containing $20 \mathrm{ng}$ template DNA, $0.25 \mathrm{mM}$ dNTPs, $0.5 \mu \mathrm{M}$ oligonucleotide primers, and $1 \mathrm{U}$ of Triple Master PCR system (Eppendorf, USA). The amplification program was as follows: $3 \mathrm{~min}$ at $94{ }^{\circ} \mathrm{C}$, followed by 15 cycles of $30 \mathrm{~s}$ at $94^{\circ} \mathrm{C}, 45 \mathrm{~s} 53^{\circ} \mathrm{C}$, and $7 \mathrm{~min}$ at $72{ }^{\circ} \mathrm{C}$. After transformation, plasmids were extracted and sequenced. Other DNA manipulations were in accordance with Sambrook et al. ${ }^{36}$ and with the manufacturer's instructions for the enzymes and materials employed.

\section{Biochemical assays}

Protein extracts were loaded on a Ni-NTA resin (QIAGEN) for affinity purification; protein concentration was determined by the Bradford method and purity checked by SDS-PAGE. Activity was measured with the pH-Stat technique on a 718 STAT TITRINO (Metrohm). Fatty acids released from different triacyl glycerols (20 $\mathrm{mM}$ in a $2 \%$ arabic gum solution) were titrated with $0.01 \mathrm{M}$ sodium hydroxide. Substrates tested were tributyrin (C4), tricaprylin (C8), and trilaurin (C12). Reaction mixtures were heated or cooled at the indicated temper- ature and measurements were started 2 min after enzyme addition to test the effect of temperature on enzyme activity. Each measurement was repeated in triplicate. For inhibition experiments with Orlistat, enzymes were incubated with the reported molar excess of inhibitor dissolved in dimethyl sulfoxide (DMSO) at room temperature for $15 \mathrm{~min}$ and then assayed for activity at $29^{\circ} \mathrm{C}$ on tricaprylin. Incubation with DMSO only was also performed as a control.

\section{Biophysical analysis}

IMAC-purified proteins were buffer-exchanged via PD10 columns (Amersham Biosciences) to replace the elution buffer with $20 \mathrm{mM}$ phosphate buffer, $\mathrm{pH}$ 7.5, and $150 \mathrm{mM} \mathrm{NaCl}$. CD and fluorescence spectra were recorded on a JASCO J815 spectropolarimeter with the following parameters. Full CD spectra were acquired at different temperatures in the far-UV region at $20 \mathrm{~nm} / \mathrm{min}$ scanning speed, $0.5 \mathrm{~nm}$ data pitch, and three accumulations per spectrum. Melting curves $\left(T_{\mathrm{m}}\right)$ were obtained by heating the sample at $1{ }^{\circ} \mathrm{C} / \mathrm{min}$ and recording the ellipticity value at $222 \mathrm{~nm}$. Fluorescence emission spectra of samples with increasing concentrations of $\mathrm{GuCl}$ were acquired in the $300-$ to $400-\mathrm{nm}$ range upon excitation at $280 \mathrm{~nm}$. DSF experiments were performed at least in triplicate on IMAC-purified enzyme solutions $(1.5 \mu \mathrm{M})$ added with $3 \times$ SYPRO Orange (Sigma), recorded on a MiniOpticon 3 (BIO-RAD) on the second channel, and $T_{\mathrm{m}}$ values were extracted from the sigmoidal part of the plot. In all experiments, buffer interference, when not negligible, was subtracted. The Prism 5 software (GraphPad Software Inc.) was used for all graphic analyses, $T_{\mathrm{m}}$ determination, and statistics.

\section{Homology modeling and MD simulations}

A structure model of the PFL was built by homology modeling using the SWISS-MODEL server, ${ }^{37}$ based on the $B$. glumae lipase structure (Protein Data Bank entry: 1TAH) as the template. The Swiss-PdbViewer ${ }^{37}$ was then used to create the mutant DE-2-4 (D2Y, H30N, R127G, R152G). Energy minimization and MD simulations of PFL and DE-2-4 lipase were performed using the GROMACS ${ }^{38}$ simulation package and the OPLS/AA force field. The proteins were solvated with TIP3P water as solvent and neutralized by adding $\mathrm{Cl}^{-}$ ions. For minimization, 5000 steps of steepest descent were performed. Both systems were equilibrated for 500 ps by simulated heating to $283 \mathrm{~K}\left(10^{\circ} \mathrm{C}\right)$. After the equilibration phase, a 5 -ns production simulation at $283 \mathrm{~K}$ was performed. Subsequently, the system was heated to $323 \mathrm{~K}\left(50^{\circ} \mathrm{C}\right)$ by $5 \mathrm{~K}$ steps. The heating in each step lasted $500 \mathrm{ps}$, followed by $1.5 \mathrm{~ns}$ of production simulation. Two independent simulations consisting of equilibration, production, and heating phases were performed for both systems. The RMSF of the backbone atoms of each residue were averaged during the last $1 \mathrm{~ns}$ of each temperature for the two independent simulations.

\section{Acknowledgements}

This work was supported by FAR (Fondo di Ateneo per la Ricerca Università Milano-Bicocca) grants to M.L. and S.M.D. A mobility grant from the 
Vigoni program to P.G.-L. is also gratefully acknowledged. A.N. acknowledges a postdoctoral fellowship of the University of Milano-Bicocca. The work on molecular simulations was supported by the Deutsche Forschungsgemeinschaft (SFB716/ C1). P.G.-L. thanks Dr. Benoit Villiers for suggesting and helping with the DSF experiment and Ann Babtie for critical reading of the manuscript.

\section{References}

1. Amitai, G., Gupta, R. D. \& Tawfik, D. S. (2007). Latent evolutionary potentials under the neutral mutational drift of an enzyme. HFSP J. 1, 67-78.

2. O'Brien, P. J. \& Herschlag, D. (1999). Catalytic promiscuity and the evolution of new enzymatic activities. Chem. Biol. 6, R91-R105.

3. Tokuriki, N. \& Tawfik, D. S. (2009). Protein dynamism and evolvability. Science, 324, 203-207.

4. Tompa, P. (2005). The interplay between structure and function in intrinsically unstructured proteins. FEBS Lett. 579, 3346-3354.

5. Wroe, R., Chan, H. S. \& Bornberg-Bauer, E. (2007). A structural model of latent evolutionary potentials underlying neutral networks in proteins. HFSP J. 1, $79-87$.

6. de Champdore, M., Staiano, M., Rossi, M. \& D'Auria, S. (2007). Proteins from extremophiles as stable tools for advanced biotechnological applications of high social interest. J. R. Soc. Interface, 4, 183-191.

7. Pikuta, E. V., Hoover, R. B. \& Tang, J. (2007). Microbial extremophiles at the limits of life. Crit. Rev. Microbiol. 33, 183-209.

8. van den Burg, B. (2003). Extremophiles as a source for novel enzymes. Curr. Opin. Microbiol. 6, 213-218.

9. D’Amico, S., Marx, J. C., Gerday, C. \& Feller, G. (2003). Activity-stability relationships in extremophilic enzymes. J. Biol. Chem. 278, 7891-7896.

10. Fields, P. A. (2001). Review: protein function at thermal extremes: balancing stability and flexibility. Comp. Biochem. Physiol., Part A: Mol. Integr. Physiol. 129, 417-431.

11. Gerday, C., Aittaleb, M., Arpigny, J. L., Baise, E., Chessa, J. P., Garsoux, G. et al. (1997). Psychrophilic enzymes: a thermodynamic challenge. Biochim. Biophys. Acta, 1342, 119-131.

12. Siddiqui, K. S. \& Cavicchioli, R. (2006). Cold-adapted enzymes. Annu. Rev. Biochem. 75, 403-433.

13. Feller, G. (2007). Life at low temperatures: is disorder the driving force? Extremophiles, 11, 211-216.

14. Roca, M., Liu, H., Messer, B. \& Warshel, A. (2007). On the relationship between thermal stability and catalytic power of enzymes. Biochemistry, 46, 15076-15088.

15. Coker, J. A. \& Brenchley, J. E. (2006). Protein engineering of a cold-active beta-galactosidase from Arthrobacter sp. SB to increase lactose hydrolysis reveals new sites affecting low temperature activity. Extremophiles, 10, 515-524.

16. Fedoy, A. E., Yang, N., Martinez, A., Leiros, H. K. \& Steen, I. H. (2007). Structural and functional properties of isocitrate dehydrogenase from the psychrophilic bacterium Desulfotalea psychrophila reveal a cold-active enzyme with an unusual high thermal stability. J. Mol. Biol. 372, 130-149.

17. Leiros, H. K., Pey, A. L., Innselset, M., Moe, E., Leiros, I., Steen, I. H. \& Martinez, A. (2007). Structure of phenylalanine hydroxylase from Colwellia psychrery- thraea $34 \mathrm{H}$, a monomeric cold active enzyme with local flexibility around the active site and high overall stability. J. Biol. Chem. 282, 21973-21986.

18. Siddiqui, K. S., Feller, G., D'Amico, S., Gerday, C., Giaquinto, L. \& Cavicchioli, R. (2005). The active site is the least stable structure in the unfolding pathway of a multidomain cold-adapted alpha-amylase. J. Bacteriol. 187, 6197-6205.

19. Bell, G. S., Russell, R. J., Connaris, H., Hough, D. W., Danson, M. J. \& Taylor, G. L. (2002). Stepwise adaptations of citrate synthase to survival at life's extremes. From psychrophile to hyperthermophile. Eur. J. Biochem. 269, 6250-6260.

20. Giver, L., Gershenson, A., Freskgard, P. O. \& Arnold, F. H. (1998). Directed evolution of a thermostable esterase. Proc. Natl Acad. Sci. USA, 95, 12809-12813.

21. Merz, A., Yee, M. C., Szadkowski, H., Pappenberger, G., Crameri, A., Stemmer, W. P. et al. (2000). Improving the catalytic activity of a thermophilic enzyme at low temperatures. Biochemistry, 39, 880-889.

22. Miyazaki, K., Wintrode, P. L., Grayling, R. A., Rubingh, D. N. \& Arnold, F. H. (2000). Directed evolution study of temperature adaptation in a psychrophilic enzyme. J. Mol. Biol. 297, 1015-1026.

23. Wintrode, P. L., Miyazaki, K. \& Arnold, F. H. (2000). Cold adaptation of a mesophilic subtilisin-like protease by laboratory evolution. J. Biol. Chem. 275, 31635-31640.

24. Tokuriki, N., Stricher, F., Serrano, L. \& Tawfik, D. S. (2008). How protein stability and new functions trade off. PLoS Comput. Biol. 4, e1000002.

25. Bloom, J. D., Labthavikul, S. T., Otey, C. R. \& Arnold, F. H. (2006). Protein stability promotes evolvability. Proc. Natl Acad. Sci. USA, 103, 5869-5874.

26. Gupta, R., Gupta, N. \& Rathi, P. (2004). Bacterial lipases: an overview of production, purification and biochemical properties. Appl. Microbiol. Biotechnol. 64, 763-781.

27. Jaeger, K. E., Dijkstra, B. W. \& Reetz, M. T. (1999). Bacterial biocatalysts: molecular biology, three-dimensional structures, and biotechnological applications of lipases. Annu. Rev. Microbiol. 53, 315-351.

28. Gatti-Lafranconi, P., Caldarazzo, S. M., Villa, A., Alberghina, L. \& Lotti, M. (2008). Unscrambling thermal stability and temperature adaptation in evolved variants of a cold-active lipase. FEBS Lett. $582,2313-2318$.

29. Alquati, C., De Gioia, L., Santarossa, G., Alberghina, L., Fantucci, P. \& Lotti, M. (2002). The cold-active lipase of Pseudomonas fragi. Heterologous expression, biochemical characterization and molecular modeling. Eur. J. Biochem. 269, 3321-3328.

30. Benjwal, S., Verma, S., Rohm, K. H. \& Gursky, O. (2006). Monitoring protein aggregation during thermal unfolding in circular dichroism experiments. Protein Sci. 15, 635-639.

31. Royer, C. A. (2006). Probing protein folding and conformational transitions with fluorescence. Chem. Rev. 106, 1769-1784.

32. Hawe, A., Sutter, M. \& Jiskoot, W. (2008). Extrinsic fluorescent dyes as tools for protein characterization. Pharm. Res. 25, 1487-1499.

33. Niesen, F. H., Berglund, H. \& Vedadi, M. (2007). The use of differential scanning fluorimetry to detect ligand interactions that promote protein stability. Nat. Protoc. 2, 2212-2221.

34. Khersonsky, O., Roodveldt, C. \& Tawfik, D. S. (2006). Enzyme promiscuity: evolutionary and mechanistic aspects. Curr. Opin. Chem. Biol. 10, 498-508. 
35. Bloom, J. D. \& Arnold, F. H. (2009). In the light of directed evolution: pathways of adaptive protein evolution. Proc. Natl Acad. Sci. USA, 106, 9995-10000.

36. Sambrook, K. J., Fritsch, E. F. \& Maniatis, T. (1989). Molecular Cloning: A Laboratory Manual, 2nd edit. Cold Spring Harbor Laboratory Press, Cold Spring Harbor, NY.
37. Guex, N. \& Peitsch, M. C. (1997). SWISS-MODEL and the Swiss-PdbViewer: an environment for comparative protein modeling. Electrophoresis, 18, 2714-2723.

38. Hess, B., Kutzner, C., van der Spoel, D. \& Lindahl, E. (2008). GROMACS 4: algorithms for highly efficient, load-balanced, and scalable molecular simulation. J. Chem. Theory Comput. 4, 435-447. 